Daraus folgen für $q$ und $\mu$ die endgültigen Werte:

$$
q=28.7 \quad \mu=3.3 .7 .
$$

Das endgültige Lösungssystem ist also:

$$
\begin{array}{ll}
q=28.7 & \mu=+33.7 \\
A=290^{\circ} 40^{\prime} & \Omega=92^{\circ} \circ^{\prime} \\
D=+27^{\circ} 10^{\prime} & \Theta=-66^{\circ} 6^{\prime}
\end{array}
$$

\begin{tabular}{|c|c|c|c|c|c|c|}
\hline & \multicolumn{2}{|c|}{$\overline{\Delta \alpha \cos \delta}$} & \multirow{2}{*}{$B-R$} & \multicolumn{2}{|c|}{$\overline{\Delta \delta}$} & \multirow{2}{*}{$B-R$} \\
\hline & $\mathrm{R}$ & $B$ & & $\mathrm{R}$ & $\mathrm{B}$ & \\
\hline 0 & +14.2 & +8.3 & -5.9 & -24.5 & -26.9 & -2.4 \\
\hline I & +15.8 & +9.5 & -6.3 & -25.8 & -27.9 & $-2 . \mathrm{I}$ \\
\hline II & +15.4 & +12.4 & -3.0 & -26.2 & -28.1 & -1.9 \\
\hline III & + 12.6 & +11.5 & - I. I & $-25 \cdot 3$ & -25.8 & -0.5 \\
\hline IV & +8.3 & +9.9 & $+\quad 1.6$ & -23.2 & -22.1 & $-\mathbf{I} . \mathbf{I}$ \\
\hline $\mathrm{V}$ & +2.9 & $-2 . I$ & -5.0 & -19.9 & $-\mathrm{x} 8.8$ & $+I$. \\
\hline VI & $-3 . I$ & - ro.9 & $-\quad 7.8$ & - I 6.7 & -16.2 & to. \\
\hline VII & $-\quad 9.2$ & $-21 \cdot 3$ & - I 2.1 & -13.9 & - 14.9 & -1.0 \\
\hline VIII & - I 6.8 & -27.9 & - I I. I & I 2.7 & $-\mathrm{I} 2.2$ & +0.5 \\
\hline IX & -24.7 & $-3^{6.1}$ & - I 2.6 & -10.5 & $-I 1.2$ & -0 \\
\hline$x$ & $-3 I .7$ & -40.7 & -9.0 & -7.8 & -7.2 & +0.6 \\
\hline $\mathrm{XI}$ & $-3^{6.5}$ & -40.9 & -4.4 & -4.8 & -3.7 & +1, \\
\hline $\mathrm{XII}$ & -39.8 & -38.2 & +1.6 & -2.4 & +2.6 & +5 \\
\hline XIII & -4 I.I & $-3^{6.1}$ & +5.0 & -0.7 & +3.9 & +4 \\
\hline XIV & -40.2 & -33.0 & +7.2 & +0.2 & +2.0 & +1 \\
\hline XV. & -37.3 & -29.2 & $+8 . \mathbf{I}$ & 0.0 & $-3 \cdot 3$ & -3 \\
\hline XVI & -33.4 & -22.0 & + II .4 & $-I . I$ & -7.7 & -6 \\
\hline XVII & -27.5 & $-\mathrm{r} 5.3$ & $+\mathrm{I} 2.2$ & -3.1 & -7.8 & -4 \\
\hline XVIII & -21.6 & -8.2 & +13.4 & $-\quad 7.9$ & -7.1 & +0 \\
\hline
\end{tabular}

Setzt man diese Werte in die Ausgangsgleichung ein und vergleicht die Resultate der Rechnung mit denen der

\begin{tabular}{|c|c|c|c|c|c|c|}
\hline & \multicolumn{2}{|c|}{$\Delta \alpha \cos \delta$} & \multirow{2}{*}{$B-R$} & \multicolumn{2}{|c|}{$\overline{\Delta \Sigma}$} & \multirow{2}{*}{$B-k$} \\
\hline & $\mathrm{R}$ & B & & $\underline{\mathrm{R}}$ & B & \\
\hline XIX & - I 5.4 & 3.2 & $+\mathrm{I} 2.2$ & -9.1 & -5.6 & $+3 \cdot 5$ \\
\hline $\mathrm{XX}$ & -8.8 & -0.6 & +8.2 & $-\mathrm{I} 2.5$ & -8.4 & +4.1 \\
\hline XXI & -2.5 & +0.9 & +3.4 & - I 5.8 & -13.7 & +2.1 \\
\hline XXII & $+4 . I$ & $+1,6$ & -2.5 & -19.3 & -20.2 & -0.9 \\
\hline XXIII & +9.6 & +5.4 & -4.2 & -22.0 & -24.7 & +2.7 \\
\hline
\end{tabular}
Beobachtung, so ergibt sich folgende Zusammenstellung:

Wien, I919 Sept. 26.
Wenn man bedenkt, daß die Sterne der Zone Berlin A, d. i. $+\mathrm{I} 5^{\circ}-20^{\circ}$, nur einen sehr geringen Teil der Sphäre bedecken, ist die Darstellung der Apexkoordinaten eine befriedigende zu nennen.

Was den Pol der Rotation anbelangt, so fällt er innerhalb der Grenzen der erreichbaren Genauigkeit mit dem Pol der Ekliptik zusammen, d. h. die gefundene Rotationsgröße $a=+33.7$ ist als Korrektur der Präzessionskonstante anzusehen.

Eine Drehung um den Pol der Milchștraße konnte aus dem vorhandenen Material nicht nachgewiesen werden.

Auffällig ist die geringe Größe der parallaktischen Bewegung: $q=28.7$, die unter der Annahme einer Sonnengeschwindigkeit von $20 \mathrm{~km}$ pro Sekunde auf eine mittlere Parallaxe aller Sterne $\pi=$ o."068 führt, während die Kapteynsche Formel $\pi=$ o." I I 7 ergibt.

Schließlich ist noch zu erwähnen, daß sich in den Resten der $\_\alpha \cos \delta$ ein deutlicher Gang zu erkennen gibt, der bei den Resten der $\mathcal{L} \delta$ nicht auftritt. Ob systematische Katalogfehler oder das Nichtzutreffen der Annahme von der Regellosigkeit der Spezialbewegungen diese Erscheinung veranlassen, kann aus dem Material einer einzigen Zone nicht entschieden werden.

G. Rickert

\title{
Beobachtungen von V $19=$ SS Cygni. (Fortsetzung von A. N. 4877.)
}

Im Jahre rq 8 erhielt ich in' 83 Nächten 84 Beobachtungen des Veränderlichen $\mathrm{V}_{\mathrm{i} 9}=\mathrm{SS}$ Cygni. Daß diese Zahl so weit gegen diejenige von sämtlichen früheren Jahren zurücksteht - im Mittel wurden in den Jahren $1905^{\circ}$ bis einschließlich I9I 7 je I 5 I Schätzungen pro Jahr gesichert liegt nicht nur an der ziemlich ungünstigen Witterung, sondern auch daran, daß mir vom 1. Juli bis Mitte September nur ein 3-zölliger Kometensucher zur Verfügung stand (s. meinen Jahresbericht über das Jahr I9 18 in der Vierteljahrsschrift der Astr. Ges., Jahrg. 54), mit welchem nur ein paar vereinzel̦te Schätzungen angestellt wurden.

Die Beobachtungsreihe weist demzufolge leider diesmal mehrere größere Lücken auf. Zwar ist mir, wie die Vergleichung mit den Ergebnissen anderer Beobachter (s. Journal of the British Astr. Ass. 29. I 24 ) lehrt, kein Maximum entgangen, aber es konnte der Typus von drei sehr unvollständig beobachteten Maxima nicht aus meinen eigenen Beobachtungen festgestellt werden.

Hier folgt eine Übersicht der Maxima des Jahres I 9 I 8.

\begin{tabular}{|c|c|c|c|c|c|c|c|c|}
\hline Ep. & $\mathrm{V}=9^{\mathrm{m}} \cdot 35$ & Maxi & mum & Gr. & $\mathrm{Bb}$. & Dauer & Typus & घี \\
\hline & & 242 & 1918 & & & & & \\
\hline 98 & 1623 & $1627=$ & Febr. 2 & $8^{m} 6$ & 6 & I $8^{\mathrm{d}}$ & lang & \\
\hline 99 & 1684.6 & I $689 ?$ & April 5? & - & 2 & -- & anormal & I \\
\hline 100 & I 745.4 & I 749 & Juni 4 & 8.5 & 6 & - & lang & \\
\hline rOI & 1809 & 18 I $2 ?$ & Aug. 6? & - & 2 & - & anormal & 1 \\
\hline 102 & - & - & 一 & - & 3 & $<$ I 4 & kurz & 2 \\
\hline I03 & 1 898? & I 904 & Nov. 6 & 8.4 & 8 & 18 & lang & \\
\hline 104 & -- & - & - . & - & 2 & - & kurz & \\
\hline
\end{tabular}
Für die Bedeutung der Kolumnen 2, 5, 6 und alle weieiteren
Einzelheiten verweise ich auf meinen Jahresbericht über das Jahr I9 I6 (AN 204.7 I).

Bemerkungen. I. Nach JBAA 29. I 24 ist das Maximum symmetrisch. - 2. Ganz unsicher. Der Stern wurde im Kometensucher am 3. September nicht gesehen, am I 5. und 16 . Sept. $=$ I $^{\mathrm{m}} .6$ geschätzt, -am 2 I. Sept. im Zehnzöller $=I_{1} \mathrm{~m}_{2}$. Nach JBAA 29.124 fing am 4. Sept. der Anstieg zu einem kurzen Maximum an. - 3. Es wurde nur der Abstieg beobachtet. Nach JBAA 29.1 24 ist das Maximum kurz. Utrecht, I9I9 Sept. I 6.
A. A. Nijland.

Brorsen-Metcalfscher Komet. Die Verbindung der Erscheinung vom Jahre 1847 mit der diesjährigen ist von Herrn cand. Duckert zur Bearbeitung übernommen. 